\title{
Electrodispersion of a liquid of finite electrical conductivity in an immiscible dielectric liquid
}

\author{
F. J. Higuera \\ E. T. S. Ingenieros Aeronáuticos, UPM Pza. Cardenal Cisneros 3, Madrid 28040, Spain
}

\begin{abstract}
Order-of-magnitude estimates and numerical computations are used to analyze an electrospray operating in the cone-jet mode in a bath of an immiscible dielectric liquid. In agreement with experimental results in the literature, the analysis predicts that the electric current carried by the jet increases as the square root of the flow rate of dispersed liquid in a wide range of conditions of the flow. The characteristics of the current transfer region determining the electric current are estimated taking into account the viscous drag of the dielectric liquid that surrounds the jet. The electric current is predicted to depart from the square root law for small flow rates, when charge relaxation effects become important in the current transfer region, and also when the flow rate increases to values of the order of $Q_{M}=\epsilon_{0} \gamma^{2} a / \mu_{2}^{2} K$, where $\epsilon_{0}$ and $\mu_{2}$ are the permittivity and viscosity of the dielectric liquid, $K$ is the electrical conductivity of the dispersed liquid, $a$ is the radius of the capillary needle through which this liquid is injected, and $\gamma$ is the interfacial tension of the liquid pair. When the flow rate becomes of order $Q_{M}$, the meniscus at the tip of the capillary ceases to resemble a Taylor cone, the current transfer region ceases to be short compared to the size of the meniscus, the electric current levels to a constant value, and the stationary jet cannot extend very far downstream of the meniscus.
\end{abstract}

\section{INTRODUCTION}

Electrodispersion of an electrically conducting liquid in an immiscible dielectric liquid is a technique to generate emulsions with narrow distributions of droplet sizes controllable in the range from micrometers to tens of nanometers. Potential applications of these emulsions include the synthesis of nanoparticles, the encapsulation of antibacterial or antifungal agents, and the manufacturing of drug-laden particles for targeted delivery.

Barrero et al. ${ }^{1}$ showed that the cone-jet mode of an electrospray, which is the simplest mode in which a conducting liquid can be electroatomized in a gas or a vacuum (see Fernández de la $\mathrm{Mora}^{2}$ for a recent review), can also be used in a liquid bath, while Alexander ${ }^{3}$ investigated the pulsating modes of an electrospray in a liquid bath. Here attention is confined to stationary cone-jets. In this mode, a meniscus of the liquid to be dispersed is formed at the end of a metallic needle through which this liquid is injected. The needle is immersed in the liquid bath, and a high voltage is applied between the needle and another electrode also immersed in the bath, leading to an electric field on the meniscus. This field induces an electric current in the conducting liquid, which accumulates electric charge at the interface between the two liquids and causes an electric stress at the interface. In appropriate ranges of the applied voltage and the flow rate injected through the needle, this stress elongates the meniscus into a cone with a thin stationary jet issuing from its apex. The jet carries an electric current which is made of the conduction current in the bulk of the conducting liquid and the convection current due to the transport of the electric charge at the interface by the flow of the liquid. Conduction is the dominant contribution to the current in the meniscus, while convection begins to play a role in a certain current transfer region and is important or dominant far downstream in the jet. The jet may break up into nearly monodisperse drops through an axisymmetric capillary instability at some distance downstream of the meniscus, or may undergo asymmetric (whipping) oscillations which end up breaking it into a more polydisperse distribution of drops.

From their measurements of the electric current/flow rate $(I-Q)$ characteristic for glycerine, ethylene-glycol and water electrosprayed in baths of hexane and heptane. Barrero et al. ${ }^{1}$ concluded that the $I \propto Q^{1 / 2}$ law, which is the hallmark of the cone-jet mode in air or vacuum, ${ }^{2,4}$ is also realized in a liquid bath. Marín ${ }^{5}$ extended these results to a wide range of liquid conductivities, confirming the square root law for moderate flow rates, though with a proportionality constant different from that of Barrero et al., and showing that the electric current falls slightly below the square root law for high flow rates. Both authors find that the minimum flow rate at which a cone-jet can be established in a liquid bath is smaller than in air and of the order of $Q_{0}=\epsilon_{0} \gamma / \rho K$, where $\rho$ and $K$ are the density and electrical conductivity of the dispersed liquid, $\gamma$ is the interfacial tension, and $\epsilon_{0}$ is the permittivity of the dielectric liquid.

Using photo and high speed video visualizations, Marín ${ }^{5}$ and Riboux et al. ${ }^{6}$ showed that electrified jets in dielectric liquid baths are more prone to whipping than their counterparts in air. Thus, these authors find that jets of glycerine in hexane undergo whipping when $Q / Q_{0}$ is higher than a certain critical value, or even for any flow rate above the minimum, which never happens for jets of glycerine in air. The distance to the meniscus at which the whipping instability develops is also smaller than in air. In additional experiments 


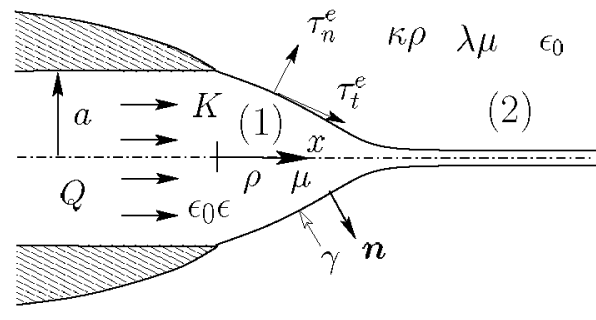

FIG. 1. Definition sketch.

with a surfactant added to the bath, Marín ${ }^{5}$ found that whipping may begin immediately downstream of the meniscus, which takes then the form of a slender cone, while the electric current carried by the jet departs from the square root law and becomes nearly independent of the flow rate. Riboux et $a l^{6}$ further characterized the whipping of the jet, showing that it occurs in a region of the flow rate/applied voltage plane bounded by a minimum flow rate and a minimum voltage which increases slightly with the flow rate. They find that the electric current increases as the power $1 / 5$ of the flow rate and as the power $4 / 3$ of the voltage, though these results were obtained mostly at high flow rates, for which the meniscus is not a Taylor cone, and attribute the new power laws to differences between the charge transport mechanisms in their jets and the jets of electrosprays in air. ${ }^{2}$

Similar results have been found by other authors. Thus, in an experiment with a cone-jet of an ionic liquid in a bath of heptane, which was a preliminary to their study of electrospraying of insulating liquids via injection of charge, Larriba and Fernández de la Mora ${ }^{7}$ found that the electric current depends weakly on the flow rate and increases faster than linearly with the voltage applied between the electrodes. They attributed these results to the space charge of the drops of the spray, which drift more slowly in heptane than in air or vacuum, and therefore screen the meniscus and the jet to an extent that increases with the flow rate and decreases with the applied voltage. Gundabala and Fernández-Nieves ${ }^{8}$ carried out experiments with an electrospray embedded in a microfluidic channel, in which a viscous oil is electrodispersed in an immiscible coflowing oil of similar viscosity. They found a stationary cone-jet regime in which the jet may break into drops through the axisymmetric capillary instability or undergo whipping, depending on the value of the flow rate. In either case, the measured current is nearly independent of the flow rate and increases linearly with the voltage. These authors explained their results in terms of the retarding force of the outer liquid on the jet and the dependence of the electric field around the tip of the meniscus on the applied voltage.

In this paper, the electrohydrodynamic problem describing the flow, the electric field, and the distribution of surface charge in an electrified stationary jet injected into a dielectric liquid bath is formulated, and a qualitative analysis is carried out to explain some of the experimental results summarized above. The radius of the jet in the current transfer region, the length of this region, and the total electric current carried by the jet are estimated for values of the flow rate above the minimum at which a cone-jet can be established. The estimate of the electric current as a function of the flow rate suggests a square root law insofar as the electric field seen by the current transfer region is that of a Taylor cone, irrespective of the relative importance of the inertia of the liquids, the force due to the axial viscous stress in the conducting liquid, and the force due to the viscous shear stress of the dielectric liquid on the jet. However, when this shear stress is important, the estimates show that a straight stationary jet ceases to be possible when the length of the current transfer region becomes of the order of the size of the meniscus, which suggests that whipping or breakup occurs then at distances from the meniscus of the order of its size. The electric current tends to a constant independent of the flow rate in these conditions. Some numerical computations of stationary jets of very viscous liquids have been carried out to back up these estimates.

\section{FORMULATION}

The configuration sketched in Fig. 1 will be considered for definiteness. A flow rate $Q$ of a liquid of density $\rho$, viscosity $\mu$, permittivity $\epsilon_{0} \epsilon$, and electrical conductivity $K$ (liquid 1 hereafter) is injected through a long metallic capillary needle into a region occupied by a dielectric liquid of density $\kappa \rho$, viscosity $\lambda \mu$, and permittivity $\epsilon_{0}$ (liquid 2), which is at rest far from the needle. The needle has the shape of a paraboloid of revolution, of mean curvature at the tip $a^{-1}$, and truncated at a distance $a / 2$ from this tip, so that the radius of the final section is $a$. A paraboloidal needle rather than the more usual tapered cylindrical needle is assumed for convenience to simplify the numerical computations of Sec. IV below by using paraboloidal coordinates. The two liquids are immiscible with an interfacial tension $\gamma$. A high voltage is applied between the needle and another far electrode perpendicular to the needle, which leads to an electric field $\boldsymbol{E}_{\infty}=-\nabla \varphi_{\infty}$, with $\varphi_{\infty}=A \ln \left\{\left[\sqrt{x^{2}+r^{2}}+x\right] / a\right\}$, at distances from the end of the needle large compared to its radius $a$ (see, e.g., Ref. 9). Here $A$ is a constant and $x$ and $r$ are distances along and normal to the axis of the needle, measured from its end.

The electric field in liquid $i$, with $i=1,2$, is $\boldsymbol{E}_{i}=-\boldsymbol{\nabla} \varphi_{i}$, where the electric potential $\varphi_{i}$ satisfies Laplace's equation. The velocity and pressure distributions, $\boldsymbol{v}_{i}$ and $p_{i}$, satisfy the continuity and Navier-Stokes equations in each liquid. The interface between the liquids, say $f(\boldsymbol{x}, t)=0$, is a material surface to be determined as part of the solution. Conduction in the inner liquid accumulates electric charge at the interface and this charge is convected by the flow leading to a surface convection current additional to the conduction current in the inner liquid. The mathematical formulation of the problem is similar to that of Ref. 10 and will be discussed only briefly here. In the cone-jet regime, the flow and the distribution of surface charge are stationary and axisymmetric in the meniscus and in a certain stretch of the jet. The interface is sought in the form $r=r_{s}(x)$ in this region of stationary flow. The governing equations are 


$$
\begin{aligned}
& \nabla^{2} \varphi_{i}=0, \quad \boldsymbol{\nabla} \cdot \boldsymbol{v}_{i}=0, \\
& \rho_{i} \boldsymbol{v}_{i} \cdot \nabla \boldsymbol{v}_{i}=-\nabla p_{i}+\mu_{i} \nabla^{2} \boldsymbol{v}_{i},
\end{aligned}
$$

in liquids 1 and 2, where $f(\boldsymbol{x})<0\left[r<r_{s}(x)\right]$ and $f(\boldsymbol{x})>0$ $\left[r>r_{s}(x)\right]$, respectively. Here $\rho_{1}=\rho, \mu_{1}=\mu, \rho_{2}=\kappa \rho$, and $\mu_{2}$ $=\lambda \mu$. These equations are to be solved with the boundary conditions

$$
\begin{aligned}
& \epsilon_{0}\left(E_{2 n}-\epsilon E_{1 n}\right)=\sigma, \quad E_{1 t}=E_{2 t}, \\
& \boldsymbol{v}_{1} \cdot \nabla \sigma=K E_{1 n}+\sigma \boldsymbol{n} \cdot \nabla \boldsymbol{v}_{1} \cdot \boldsymbol{n}, \\
& \boldsymbol{v}_{1} \cdot \nabla f=0, \quad \boldsymbol{v}_{1}=\boldsymbol{v}_{2}, \\
& p_{2}-p_{1}+\boldsymbol{n} \cdot\left(\tau_{1}^{\prime}-\tau_{2}^{\prime}\right) \cdot \boldsymbol{n}+\gamma \nabla \cdot \boldsymbol{n}=\tau_{n}^{e}, \\
& \boldsymbol{t} \cdot\left(\tau_{1}^{\prime}-\tau_{2}^{\prime}\right) \cdot \boldsymbol{n}=\tau_{t}^{e},
\end{aligned}
$$

at the interface, $f(\boldsymbol{x})=0$, which is assumed to be attached to the end of the needle $\left[r_{s}(0)=a\right]$,

$$
\boldsymbol{v}_{1}=\frac{2 Q}{\pi a^{2}}\left(1-\frac{r^{2}}{a^{2}}\right) \boldsymbol{i}
$$

at the outlet, $x=0, r<a$,

$$
\boldsymbol{v}_{2}=0 \text {, }
$$

at the outer surface of the needle, $r=r_{p}(x)=a(1-2 x / a)^{1 / 2}$ for $x<0$, and

$$
\begin{aligned}
& p_{2}=0, \quad \boldsymbol{v}_{2}=0, \\
& \varphi_{1} \sim \varphi_{2} \sim \varphi_{\infty}=A \ln \frac{\sqrt{x^{2}+r^{2}}+x}{a},
\end{aligned}
$$

far from the needle.

Here $p_{i}$ is the pressure of liquid $i$ referred to the pressure of the outer liquid far from the needle, $\boldsymbol{n}=\boldsymbol{\nabla} f /|\boldsymbol{\nabla} f|$ is a unit vector normal to the interface pointing toward the outer liquid and $t$ is a unit vector tangent to the interface in a meridional section, $E_{i n}=\boldsymbol{E}_{i} \cdot \boldsymbol{n}$ and $E_{i t}=\boldsymbol{E}_{i} \cdot \boldsymbol{t}$ are the components of the electric field in liquid $i$ normal and tangent to the interface, $\tau_{i}^{\prime}=\mu_{i}\left[\nabla \boldsymbol{v}_{i}+\left(\nabla \boldsymbol{v}_{i}\right)^{T}\right]$ is the viscous stress tensor of liquid $i, \sigma$ is the density of free surface charge, $\boldsymbol{i}$ is a unit vector in the direction of the axis of the needle, and $\tau_{n}^{e}$ and $\tau_{t}^{e}$ in the right-hand sides of Eqs. (5) and (6) are the electric stresses normal and tangent to the interface, given by (see Landau and Lifshitz ${ }^{11}$ and Saville ${ }^{12}$ )

$$
\begin{aligned}
\tau_{n}^{e} & =\frac{\epsilon_{0}}{2}\left(E_{2 n}^{2}-\epsilon E_{1 n}^{2}\right)+\frac{\epsilon_{0}}{2}(\epsilon-1) E_{1 t}^{2}, \\
\tau_{t}^{e} & =\sigma E_{1 t} .
\end{aligned}
$$

In Eq. (2) are electrostatic conditions for the electric field at the interface; see, e.g., Landau and Lifshitz. ${ }^{11}$ Equation (3) is the transport equation for the free surface charge. The first term on the right-hand side of this equation is the rate of accumulation of charge per unit area of the interface due to the component of the conduction current density in liquid $1, \boldsymbol{j}=\boldsymbol{K} \boldsymbol{E}_{1}$, normal to the interface. The second term is the rate of change of $\sigma$ due to the stretching of the interface (see, e.g., Batchelor ${ }^{13}$ ). Equations (5) and (6) are balances of stresses normal and tangent to the interface; the terms on the left-hand side of Eq. (5) are the normal stresses due to pressure, viscosity, and surface tension. Equation (7), stating that the velocity distribution of the inner liquid is parabolic at the end of the needle, is a simplification of the real flow in the needle. The approximation is not expected to have an important effect on the solution, given the strong stretching of the jet under the action of the electric stresses at the interface.

In the jet, where the flow of the inner liquid is quasiunidirectional with a velocity nearly uniform in each crosssection, of the form $\boldsymbol{v}_{1} \approx v(x) \boldsymbol{i}$ with $\pi r_{s}^{2} v=Q$, the third Eq. (1) can be integrated to give (see, e.g., Gañán-Calvo et al. ${ }^{14}$ and Feng ${ }^{15}$ )

$$
\begin{aligned}
\frac{\partial}{\partial x}\left(\pi r_{s}^{2} \rho v^{2}\right)= & \frac{\partial}{\partial x}\left(3 \pi r_{s}^{2} \mu \frac{\partial v}{\partial x}\right)+\pi r_{s}^{2} \frac{\partial}{\partial x}\left(\tau_{n}^{e}+\tau_{n}^{o}-\frac{\gamma}{r_{s}}\right) \\
& +2 \pi r_{s}\left(\tau_{t}^{e}-\tau_{t}^{o}\right),
\end{aligned}
$$

where $\tau_{n}^{o}=-p_{2}+\boldsymbol{n} \cdot \tau_{2}^{\prime} \cdot \boldsymbol{n}$ and $\tau_{t}^{o}=-\boldsymbol{t} \cdot \boldsymbol{\tau}_{2}^{\prime} \cdot \boldsymbol{n}$ are the pressure and viscous stresses of the outer liquid normal and tangent to the interface.

\section{ORDER OF MAGNITUDE ESTIMATES}

\section{A. Electrical estimates}

This section summarizes well-known estimates of the electric field and the surface charge at the beginning of the jet. In cases when the radius of the jet issuing from the tip of the meniscus is small compared to the radius $a$ of the injection orifice, the balance of surface tension and normal electric stress (of order $\epsilon_{0} E_{2 n}^{2}$ ) requires that the meniscus be nearly an equipotential cone (Taylor ${ }^{16}$ ) at distances from its tip small compared to $a$ but large compared to the radius of the jet. The electric field in the outer liquid is $E_{T}$ $\sim\left(\gamma / \epsilon_{0} x^{\prime}\right)^{1 / 2}$ in this region, where $x^{\prime}$ is the distance to the apparent vertex of the cone.

The jet is immersed in the electric field $E_{T}$, at least at distances from the apparent vertex small compared to $a$. Since the inner liquid is an electric conductor, the electric field carries charge to the interface, where it accumulates and tends to screen this liquid from the field $E_{T}$. Electrically, the jet acts as a line of charge that induces an axial field of order $E_{2 n} r_{s} / x^{\prime}$ up to a logarithmic factor $\left(\right.$ Hinch $^{17}$ ), where $E_{2 n}$ is the field normal to the jet at the outer side of the interface. The condition

$$
E_{2 n} \frac{r_{s}}{x^{\prime}} \sim E_{T}
$$

is satisfied in the region of the jet where the induced axial field is able to balance the outer field $E_{T}$. The length of this region is estimated in the following paragraph.

The density of free surface charge needed to generate a normal field $E_{2 n}$ is $\sigma \approx \epsilon_{0} E_{2 n}$ when the effect of the electric displacement in the inner liquid is negligible in the first Eq. (2); see comments at the end of this section. This surface 
charge is convected by the flow, leading to a surface current $I_{s}=2 \pi \sigma v r_{s}$, and must therefore be continuously replaced by new charge carried to the interface by radial conduction in the inner liquid. The balance of charge per unit length of the jet reads $\mathrm{d} I_{s} / \mathrm{d} x=2 \pi r_{s} K E_{1 n}$, which is an integrated form of Eq. (3). In orders of magnitude, this balance requires $I_{s} / x^{\prime} \sim r_{s} K E_{1 n}$ or $\epsilon_{0} E_{T} Q / r_{s}^{2} \sim r_{s} K E_{1 n}$, where use has been made of $\sigma \sim \epsilon_{0} E_{2 n}$, Eq. (12), and the equality $v=Q / \pi r_{s}^{2}$. Using this result and the condition $\boldsymbol{\nabla} \cdot \boldsymbol{E}_{1}=0$ (which amounts to $\left.E_{1 n} / r_{s} \sim E_{1 x} / x^{\prime}\right)$, the axial field in the inner liquid can be estimated as $E_{1 x} \sim \epsilon_{0} E_{T} Q x^{\prime} / K r_{s}^{4}$. This field becomes of the order of $E_{T}$ for

$$
\epsilon_{0} Q x^{\prime} \sim K r_{s}^{4},
$$

which defines the end of the region where the surface charge can keep the field $E_{T}$ out of the inner liquid.

The estimates leading to Eq. (13) also imply that the surface current $I_{s}$ is of the order of the conduction current in the inner liquid, $I_{b}=2 \pi K \int_{0}^{r_{s}} E_{1 x} r \mathrm{~d} r$, in the region where $E_{1 x}$ $\sim E_{T}$ for the first time (because $I_{b} \sim r_{s}^{2} K E_{T} \sim r_{s} K E_{1 n} x^{\prime} \sim I_{s}$ ). Therefore this region can be identified with the current transfer region where convection of the surface charge begins to account for a substantial part of the current of the jet.

The electric charge in the surface of the current transfer region is $\mathcal{C}=\int 2 \pi r_{s} \sigma \mathrm{d} x^{\prime} \sim \sigma r_{s} x^{\prime} \sim I_{s} t_{r}$, where $t_{r}=x^{\prime} / v$ is the residence time of the inner liquid in this region. Since $I_{s} \sim I_{b}$, this time is of the order of the time $\mathcal{C} / I_{b}$ required for conduction to supply the charge $\mathcal{C}$ to the surface. On the other hand, the time required for the density of surface charge to attain its equilibrium value $\epsilon_{0} E_{2 n}$ and screen the inner liquid is the electric relaxation time $t_{e}=\epsilon_{0} \epsilon / K$ [from the order-of-magnitude balance $\sigma / t_{e} \sim K E_{1 n}$ with $\sigma \sim \epsilon_{0} E_{2 n}$ $\sim \epsilon_{0} \in E_{1 n}$; see Eqs. (2) and (3)]. Therefore the condition $t_{r} \gg t_{e}$ must be satisfied for the condition $\sigma \approx \epsilon_{0} E_{2 n}$ used above to be satisfied.

Using the definitions of $t_{r}$ and $t_{e}$, Eq. (13) can be rewritten as $x^{\prime} / r_{s} \sim \epsilon^{1 / 2}\left(t_{r} / t_{e}\right)^{1 / 2}$, which means that the aspect ratio $x^{\prime} / r_{s}$ of the current transfer region is large compared to $\epsilon^{1 / 2}$ when $t_{r} \gg t_{e}$ and becomes of order $\epsilon^{1 / 2}$ when charge relaxation effects become important.

\section{B. Mechanical estimates}

The electric stress normal to the interface is $\tau_{n}^{e} \sim \epsilon_{0} E_{n}^{2}$ $\sim \epsilon_{0} E_{T}^{2}\left(x^{\prime} / r_{s}\right)^{2}$ in the region where Eq. (12) applies. The axial gradient of the depression induced in the inner liquid by this stress gives rise to the force $\pi r_{s}^{2} \partial \tau_{n}^{e} / \partial x \sim \epsilon_{0} E_{T}^{2} x^{\prime}$ in Eq. (11). This is the main force driving the inner liquid at the beginning of the jet, where the electric field tangent to the interface is small compared to $E_{T}$ and the axial force due to the electric shear stress is therefore $2 \pi r_{s} \tau_{t}^{e} \ll \epsilon_{0} E_{T}^{2} x^{\prime}$. However, the force due to the electric shear stress becomes of order $\epsilon_{0} E_{T}^{2} x^{\prime}$ and overcomes the previous force in the current transfer region.

These driving forces may (a) cause an acceleration of the inner liquid [left-hand side of Eq. (11)], or (b) be balanced by the axial viscous force due to the axial stretching of the jet [first term on the right-hand side of Eq. (11)], or (c) be balanced by the axial force due to the viscous shear stress of the outer liquid [2 $\pi r_{s} \tau_{t}^{\rho}$ in the last term of Eq. (11)]. The effect of the surface tension in the region of quasiunidirectional flow downstream of the cone-to-jet transition region is small for sufficiently high flow rates; see, e.g., Ref. 18.

The first two possibilities have been discussed elsewhere (Gañán-Calvo et al. $^{14}$ and Higuera ${ }^{18,19}$ ). Briefly summarized, the inertia/driving force balance of case (a) and the axial viscous force/driving force balance of case (b) lead to the order-of-magnitude estimates $\rho r_{s}^{2} v^{2} / x^{\prime} \sim \epsilon_{0} E_{T}^{2} x^{\prime}$ and $\mu r_{s}^{2} v / x^{\prime 2} \sim \epsilon_{0} E_{T}^{2} x^{\prime}$, respectively. Together with Eq. (12) and $E_{T} \sim\left(\gamma / \epsilon_{0} x^{\prime}\right)^{1 / 2}$, these estimates give $r_{s} \sim\left(\rho Q^{2} / \gamma x^{\prime}\right)^{1 / 2}$ in case (a) and $x^{\prime} \sim(\mu Q / \gamma)^{1 / 2}$ in case (b). Carrying these results to Eq. (13), which defines the current transfer region where $I_{s} \sim I_{b}$, we find, in this region,

$$
\begin{aligned}
& x_{c t}^{\prime} \sim x^{(a)}=\frac{\rho^{2 / 3} K^{1 / 3} Q}{\epsilon_{0}^{1 / 3} \gamma^{2 / 3}}, \\
& r_{s_{c t}} \sim r_{s}^{(a)}=\frac{\rho^{1 / 6} \epsilon_{0}^{1 / 6} Q^{1 / 2}}{K^{1 / 6} \gamma^{1 / 6}}, \\
& I \sim \gamma^{1 / 2} K^{1 / 2} Q^{1 / 2},
\end{aligned}
$$

in case (a) and

$$
\begin{aligned}
& x_{c t}^{\prime} \sim x^{(b)}=\frac{\mu^{1 / 2} Q^{1 / 2}}{\gamma^{1 / 2}}, \\
& r_{s_{c t}} \sim r_{s}^{(b)}=\frac{\epsilon_{0}^{1 / 4} \mu^{1 / 8} Q^{3 / 8}}{K^{1 / 4} \gamma^{1 / 8}}, \\
& I \sim \gamma^{1 / 2} K^{1 / 2} Q^{1 / 2},
\end{aligned}
$$

in case (b). Here the subscript $c t$ denotes conditions in the current transfer region. The estimates (14a) are valid for values of $R=\rho^{4 / 3} K^{2 / 3} Q /\left(\epsilon_{0}^{2 / 3} \mu \gamma^{1 / 3}\right)$ of order unity or large, while (14b) are valid for small values of $R$. The estimate of the current/flow rate characteristic is the same in the two cases. Here $I=I_{s}+I_{b}$ is the total electric current carried by the jet.

The shear stress of the outer liquid on the interface can be estimated as $\tau_{t}^{D} \sim \lambda \mu v / r_{s}$, up to logarithmic factors, insofar as the motion of the jet is transmitted by viscous stresses to a layer of the outer liquid of thickness of $O\left(r_{s}\right)$ or larger (Glauert and Lighthill ${ }^{20}$ ). The shear stress due to the outer liquid comes into play in the current transfer region when $2 \pi r_{s} \tau_{t}^{o} \sim \pi r_{s}^{2} \partial \tau_{n}^{e} / \partial x$ in Eq. (11), which happens when $\Pi=\lambda \mu K^{1 / 3} /\left(\rho \epsilon_{0} \gamma^{2}\right)^{1 / 3}=O(1)$ [in case (a)] or $\lambda\left(\mu^{3} K^{2} Q / \gamma^{3} \epsilon_{0}^{2}\right)^{1 / 4}=\Pi R^{1 / 4}=O(1)$ [in case (b)]. The effect of the outer liquid dominates those of the inertia and the axial viscous force of the inner liquid when the smallest of $\Pi$ and $\Pi R^{1 / 4}$ becomes large compared to unity. The relevant balance of forces in Eq. (11) for this case (c) is therefore 
$2 \pi r_{s} \tau_{t}^{0} \sim \pi r_{s}^{2} \partial \tau_{n}^{e} / \partial x$, or $\lambda \mu v \sim \epsilon_{0} E_{T}^{2} x^{\prime}$, which, together with Eq. (12) and $E_{T} \sim\left(\gamma / \epsilon_{0} x^{\prime}\right)^{1 / 2}$, gives $r_{s} \sim(\lambda \mu Q / \gamma)^{1 / 2}$, independent of $x^{\prime}$. Carrying this result to Eq. (13) we find

$$
\begin{aligned}
& x_{c t}^{\prime} \sim x^{(c)}=\frac{\lambda^{2} \mu^{2} K Q}{\epsilon_{0} \gamma^{2}}, \\
& r_{s_{c t}} \sim r_{s}^{(c)}=\frac{\lambda^{1 / 2} \mu^{1 / 2} Q^{1 / 2}}{\gamma^{1 / 2}}, \\
& I \sim \gamma^{1 / 2} K^{1 / 2} Q^{1 / 2},
\end{aligned}
$$

in case (c). The estimates of the current/flow rate characteristic coincide in all three cases; the well-known square root law appears thus as a robust result. This is in line with the findings of Barrero et al., ${ }^{1}$ who experimentally retrieved this law for all the liquid couples and operating conditions they tested. The result also means that measurements of the flow rate and electric current alone cannot discriminate between the possible regimes of the flow. Case (c) is probably realized in the experiments of Gundabala and Fernández-Nieves, ${ }^{8}$ though not in the experiments of Riboux et al. ${ }^{6}$ where $R$ and $\Pi$ are small numbers.

The length of the current transfer region and the radius of the jet in this region are larger in case (c) than in cases (a) and (b). Thus, the ratio of the estimates of $x_{c t}^{\prime}$ in Eqs. (14c) and (14a) is $\Pi^{2}$, and the ratio of the estimates in Eqs. (14c) and (14b) is $\Pi^{2} R^{1 / 2}$, both of which are large numbers when the balance of forces of case (c) is realized. The $1 / 2$ or $3 / 8$ power dependences of the radius of the jet on the flow rate are not far from the power 0.578 found experimentally by Marín $^{5}$ for jets of glycerine in hexane.

The surface current makes an important contribution to the total current of the jet for $x^{\prime} \gg x_{c t}^{\prime}$, where the condition $I_{s} \sim I$ implies $\sigma \sim I / v r_{s} \sim I r_{s} / Q$. In the presence of an electric field $E_{\text {far }}\left(x^{\prime}\right)$, this surface charge leads to an electric shear stress $\tau_{t}^{e} \sim I r_{s} E_{\mathrm{far}} / Q$ and to an axial force $2 \pi r_{s} \tau_{t}^{e}$ $\sim I_{s}^{2} E_{\mathrm{far}} / Q$ in Eq. (11). This is the main force driving the flow in the far jet and it is to be balanced by an acceleration of the liquid, or by the axial viscous force due to the stretching of the jet, or by the axial force due to the viscous shear stress of the outer liquid, as in (a) to (c) above. The relevant balances in the momentum (11), analogous to the ones discussed above, give $r_{s} \sim\left(\rho Q^{3} / I E_{\mathrm{far}} x\right)^{1 / 4}$, or $r_{s} \sim\left(\mu Q^{2} / I E_{\mathrm{far}} x^{2}\right)^{1 / 2}$, or $r_{s} \sim\left(\lambda \mu Q^{2} / I E_{\mathrm{far}}\right)^{1 / 4}$ for the radius of the far jet in each of the three possible cases. Thus, for $x_{c t}^{\prime} \ll x^{\prime} \ll a$, where $E_{\text {far }} \approx E_{T}$, these estimates give $r_{s} \sim r_{s}^{(a)} /\left(x^{\prime} / x^{(a)}\right)^{1 / 8}, \quad$ or $\quad r_{s} \sim r_{s}^{(b)} /\left(x^{\prime} / x^{(b)}\right)^{3 / 4}, \quad$ or $\quad r_{s} \sim r_{s}^{(c)}$ $\times\left(x^{\prime} / x^{(c)}\right)^{1 / 8}$. If the jet extends to distances large compared to $a$ but still small compared to the interelectrode distance, then $E_{\mathrm{far}}=E_{\infty} \approx A / x^{\prime}$ should be used in the estimates of $r_{s}$. Finally, a constant $E_{\text {far }}$ is a suitable approximation to the field farther from the needle in the needle-plate electrode configuration of Refs. 5 and 6 . In agreement with the experimental findings of Riboux et al., ${ }^{6}$ the radius of the jet and the conduction current are then independent of streamwise distance when the viscous shear stress of the outer liquid balances the electric shear stress.

\section{Small flow rates}

The ratio $t_{r} / t_{e}$ of the residence time in the current transfer region to the electric relaxation time of the inner liquid is an increasing function of the flow rate in the three cases of the previous section. The condition $t_{r} / t_{e}=O(1)$ gives the order of the flow rate for which charge relaxation effects come into play in the current transfer region, and below which Eqs. (14a)-(14c) ceases to be applicable. This characteristic flow rate is $Q_{m}^{(a)}=\epsilon Q_{0}$ with $Q_{0}=\epsilon_{0} \gamma / \rho K$ in case (a), $Q_{m}^{(b)}=\epsilon^{4} \epsilon_{0}^{2} \gamma^{3} / \mu^{3} K^{2}$ in case (b), and $Q_{m}^{(c)}=\epsilon \epsilon_{0}^{2} \gamma^{3} / \lambda^{3} \mu^{3} K^{2}$ in case (c). In case (a), charge relaxation effects come into play simultaneously in the current transfer region and in the coneto-jet transition region around the tip of the meniscus, ${ }^{21}$ and $Q_{m}^{(a)}$ is often of the order of the experimental minimum flow rate at which a cone-jet can be established ${ }^{4}$ [but see Ref. 21 and Eq. (15a) below for a possible low flow rate regime of very polar liquids with $Q \ll Q_{m}^{(a)}$ and dominant relaxation effects]. In cases (b) and (c), charge relaxation effects are negligible in the cone-to-jet transition region for values of $Q$ of the order of $Q_{m}^{(b)}$ or $Q_{m}^{(c)}$, at least if the inner liquid is very polar $\left(\epsilon\right.$ large). The ratio $Q_{m}^{(b)} / Q_{m}^{(c)}=(\lambda \epsilon)^{3}$ while $\Pi R^{1 / 4}=\lambda \epsilon$ for $Q=Q_{m}^{(b)}$ and $\Pi R^{1 / 4}=(\lambda \epsilon)^{1 / 4}$ for $Q=Q_{m}^{(c)}$. This means that charge relaxation effects appear first for $Q \sim Q_{m}^{(b)}$ when $\lambda \epsilon \ll 1$, and for $Q \sim Q_{m}^{(c)}$ when $\lambda \epsilon \gg 1$. The effect of the axial force due to the shear stress of the outer liquid is negligible in the first conditions and dominant in the second.

Low flow rate regimes for very polar liquids analogous to that of Ref. 21 for case (a) are possible also for cases (b) and (c) and are briefly discussed here for completeness. Condition (12) is valid for such regimes, with the normal field $E_{2 n}$ induced by the polarization of the inner liquid rather than by the free charge at the interface. Condition (13) is no longer valid and must be replaced by the condition $t_{r} \sim t_{e}$ (or, equivalently, $x^{\prime} / r_{s} \sim \epsilon^{1 / 2}$ ) discussed above. In combination with the balances of forces of Sec. III B, these conditions give

$$
\begin{aligned}
& x_{c t}^{\prime} \sim x^{(a)} \mid Q_{m}^{(a)}\left(\frac{Q}{Q_{m}^{(a)}}\right)^{2 / 3}, \\
& r_{s_{c t}} \sim \frac{x_{c t}^{\prime}}{\epsilon^{1 / 2}}, \\
& I \sim\left(\gamma K Q_{m}^{(a)}\right)^{1 / 2} \frac{Q}{Q_{m}^{(a)}},
\end{aligned}
$$

in case (a), for $Q \ll Q_{m}^{(a)}$, where $\left.x^{(a)}\right|_{Q_{m}^{(a)}}$ is $x_{c t}^{\prime}$ in (14a) evaluated for $Q=Q_{m}^{(a)}$,

$$
\begin{aligned}
& x_{c t}^{\prime} \sim x^{(b)}, \\
& r_{s_{c t}} \sim \frac{x_{c t}^{\prime}}{\epsilon^{1 / 2}}, \\
& I \sim\left(\gamma K Q_{m}^{(b)}\right)^{1 / 2}\left(\frac{Q}{Q_{m}^{(b)}}\right)^{3 / 4},
\end{aligned}
$$

in case (b), for $Q \ll Q_{m}^{(b)}$, and 


$$
\begin{aligned}
& x_{c t}^{\prime} \sim \epsilon^{1 / 2} r_{s}^{(c)}, \\
& r_{s_{c t}} \sim r_{s}^{(c)}, \\
& I \sim\left(\gamma K Q_{m}^{(c)}\right)^{1 / 2}\left(\frac{Q}{Q_{m}^{(c)}}\right)^{3 / 4},
\end{aligned}
$$

in case (c), for $Q \ll Q_{m}^{(c)}$.

\section{Modifications for high flow rates}

The estimates (14a)-(14c) also break down when $x_{c t}^{\prime}$ ceases to be small compared to the size of the meniscus, of order $a$, because the electric field acting on the jet ceases to be that of a conical meniscus, $E_{T}$. This happens in case (c) when $Q$ increases to values of order $Q_{M}=\epsilon_{0} \gamma^{2} a / \lambda^{2} \mu^{2} K$. If the current transfer region was to extend to distances in the jet large compared to $a$, then the field $E_{T}$ should be replaced by the field of a paraboloidal electrode, $E_{\infty} \approx A / x$. When this change is made in Eq. (12), the normal electric stress becomes $\tau_{n}^{e} \sim \epsilon_{0} A^{2} / r_{s}^{2}$, and the balance of forces characteristic of case (c), $2 \pi r_{s} \tau_{t}^{o} \sim \pi r_{s}^{2} \partial \tau_{n}^{e} / \partial x$, gives the modified estimate $r_{s} \sim\left(\lambda \mu Q x / \epsilon_{0} A^{2}\right)^{1 / 2}$ for $x \geqslant a$ but still upstream of the current transfer region. However, this result is meaningless because a $r_{s}$ increasing with $x$ leads to a driving force pointing toward the capillary needle, $\pi r_{s}^{2} \partial \tau_{n}^{e} / \partial x<0$, which cannot push the inner liquid into the jet. This negative result suggests that a straight stationary jet cannot exist at distances from the needle large compared to $a$ in the conditions of case (c), when the viscous stress of the outer liquid is important, if $Q \gg Q_{M}$. This agrees with the observations of Marín ${ }^{5}$ that the long straight jet generated when glycerine is electrosprayed in air becomes much shorter and soon undergoes asymmetric whipping oscillations when glycerine is electrosprayed in a bath of hexane.

The whipping of the jet, or its direct breakup into drops for liquids of smaller viscosity, may imply a cutoff for the transfer of electric current to its surface, which would affect the total current carried by the jet when the flow rate is of the order of $Q_{M}$ or larger. The current of the jet would then be only the current that can be transferred to its surface at distances of order $a$, before whipping or breakup prevent further transfer. This current can be estimated noticing that the surface of the jet is nearly equipotential at these distances from the meniscus when $Q \gg Q_{M}$ and the balance of forces for case (c), together with Eq. (12) and $E_{T} \sim\left(\gamma / \epsilon_{0} a\right)^{1 / 2}$, give $r_{s} \sim(\lambda \mu Q / \gamma)^{1 / 2}$ (as before) and $\sigma \approx \epsilon_{0} E_{2 n} \sim\left(\epsilon_{0} \gamma^{3} a / \lambda \mu Q\right)^{1 / 2}$. The surface convection current in this region is $I_{s} \sim \sigma v r_{s}$ $\sim \epsilon_{0}^{1 / 2} \gamma^{3 / 2} a^{1 / 2} / \lambda \mu$, independent of $Q$, which justifies some of the experimental results discussed in the introduction. ${ }^{8}$

\section{NUMERICAL RESULTS}

Some of the qualitative estimations of the previous section can be compared to numerical solutions of Eqs. (1)-(10) for very viscous liquids. The computations described below are aimed at determining the current/flow rate characteristic and the size of the current transfer region, verifying the transition from case (b) to case (c) when the flow rate is increased, and showing the existence of a maximum flow rate when the viscous shear stress of the outer liquid plays a role, as well as the conditions of the flow when this maximum is approached. The numerical results also show that charge relaxation effects become important at low flow rates, though no attempt is made to check the low flow rate regime for very polar liquids mentioned at the end of Sec. III C.

Problems (1)-(10) can be written in dimensionless form by scaling distances with the radius of the injection orifice $a$, velocities with the viscous-capillary velocity $v_{c}=\gamma / \mu$, and electric fields with $E_{c}=\left(\gamma / \epsilon_{0} a\right)^{1 / 2}$. The electric potentials, density of surface charge, and electric current are then scaled with $E_{c} a, \epsilon_{0} E_{c}$, and $\epsilon_{0} E_{c} v_{c} a$, respectively, and the pressure and viscous stresses with $\mu v_{c} / a$. The dimensionless problem depends on the seven dimensionless parameters

$$
\begin{aligned}
& \mathrm{Ca}=\frac{\mu Q}{\gamma a^{2}}, \quad V_{\infty}=\frac{\epsilon_{0}^{1 / 2} A}{\gamma^{1 / 2} a^{1 / 2}}, \quad \epsilon, \\
& \Lambda=\frac{\mu K a}{\epsilon_{0} \gamma}, \quad \lambda, \quad \operatorname{Re}=\frac{\rho \gamma a}{\mu^{2}}, \quad \kappa .
\end{aligned}
$$

No attempt will be made to explore the full parameter space of the problem. Instead, a few numerical simulations will be carried out to illustrate the features of the solution mentioned above. The inertia of both liquids will be left out considering the limit $\operatorname{Re} \rightarrow 0$ with finite values of the other parameters in (16). The value of Re may be small for very viscous liquids; for example it is $\mathrm{Re}=7 \times 10^{-4}$ for glycerine in hexane with $a=0.5 \mathrm{~mm}$. In cases when Re is not so small, the effect of the inertia of the inner liquid may be expected to be important in the meniscus and a leading region of the jet, but the left-hand side of Eq. (11) should become negligible farther in the jet when an outer liquid is present. Similarly, the inertia of the outer liquid is not expected to qualitatively change the viscous shear stress of this liquid on the interface, except perhaps in some region where a boundary layer thin compared to the radius of the jet exists in the outer liquid.

Numerical results will be presented for $\epsilon=20, \Lambda=10^{3}$, and different values of $V_{\infty}$ and $\lambda$. This value of $\Lambda$ is not far from the range $\Lambda=430-615$ covered in the experiments of Riboux et $a l^{6}$ and is somewhat larger than the value $\Lambda \approx 60$ of Gundabala and Fernández-Nieves. ${ }^{8}$ For glycerine in the conditions mentioned above, for which $\epsilon=22.75$, the value $\Lambda=10^{3}$ is attained with a conductivity $K=4$ $\times 10^{-7} \mathrm{~S} / \mathrm{m}$. The dimensionless voltage $V_{\infty}$ is bound to take values of $O(1)$ in order for a cone-jet to exist for small values of $\mathrm{Ca}$. Finally, values of the viscosity ratio $\lambda$ in the order of a few hundredths have been chosen to illustrate the transition from case (b), in which the effect of the viscous shear stress of the outer liquid is negligible in the current transfer region, to case (c), in which this effect is dominant. These values of $\lambda$ are somewhat smaller than in Ref. 8 (where $\lambda$ $\approx 0.6$ ) and substantially larger than the hexane-to-glycerine viscosity ratio, $\lambda=2.3 \times 10^{-4}$, in the experiments of Ref. 6 . The effect of the viscous shear stress of the outer liquid is small in the latter experiments.

Stationary axisymmetric solutions of the problem have been computed using boundary integral methods to solve the Laplace's equations for the electric potentials and the Stokes 

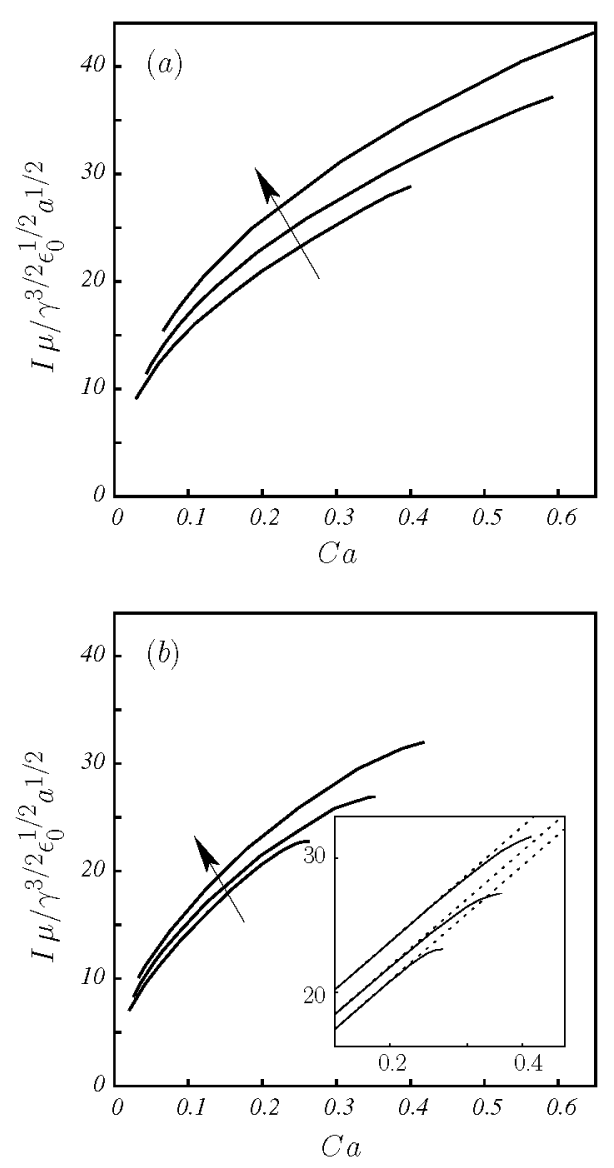

FIG. 2. Current/flow rate characteristic for $\lambda=0.01$ (a) and 0.03 (b), with $V_{\infty}=1.6,1.8$, and 2 , increasing from bottom to top as indicated by the arrows. Values of other parameters are $\epsilon=20, \Lambda=10^{3}$, and $\mathrm{Re}=0$. The inset in panel (b) shows the departure from the square root law in the vicinity of the maximum flow rate. Dotted lines have a slope of $1 / 2$.

equations that result when the inertia terms $\rho_{i} \boldsymbol{v}_{i} \cdot \boldsymbol{\nabla} \boldsymbol{v}_{i}$ are left out of Eq. (1). Transient forms of the transport equation for the surface charge (3) and the first Eq. (4) for the interface, with terms $\partial \sigma / \partial t$ and $\partial f / \partial t$ added to their left-hand sides, are marched in time until the solution converges to a stationary state. Since the breakup of the jet is not realistically described by these computations, a condition must be given for the jet at the downstream boundary of the computational domain. Here, as in previous works (see, e.g., Refs. 19 and 21), the jet is artificially truncated at a distance from the needle chosen to ensure that the solution in the region of interest is not sensitive to this truncation.

Figure 2 shows some computed electric current/flow rate characteristics. In all the cases displayed, the electric current increases as the square root of the dimensionless flow rate $\mathrm{Ca}$ for moderate values of this variable and falls below the $I \propto \mathrm{Ca}^{1 / 2}$ law for large flow rates, until a certain $\mathrm{Ca}$ is reached above which the computations cease to converge to a stationary solution. The departure from the square root law is more pronounced and occurs earlier for larger values of $\lambda$, for which the current levels off to a constant value before a stationary solution ceases to exist. This can be seen in the inset of Fig. 2(b), which is a logarithmic plot of the vicinity of the maximum flow rate for $\lambda=0.03$.

The electric current in Fig. 2 increases mildly with the

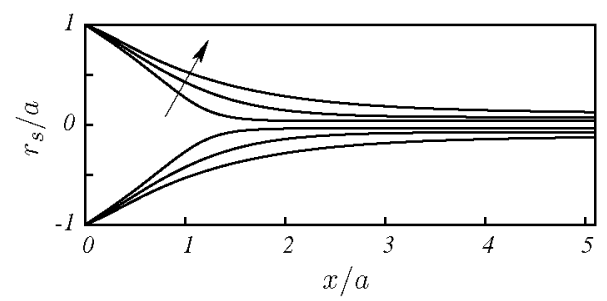

FIG. 3. Contours of the meniscus and the jet for $V_{\infty}=1.8, \epsilon=20, \Lambda=10^{3}$, $\lambda=0.03, \mathrm{Re}=0$, and $\mathrm{Ca}=0.0256,0.125$, and 0.351 , increasing as indicated by the arrow.

applied voltage $V_{\infty}$. It should be noted that these computations do not take into account the effect of the space charge of the drops far from the meniscus, which may decrease the value of $V_{\infty}$ seen by the current transfer region when the flow rate is increased keeping constant the voltage between the electrodes. As pointed out by Larriba and Fernández de la Mora, ${ }^{7}$ this space charge can moderate the increase of the electric current with the flow rate and lead to a nearly constant current in a range of flow rates wider than in Fig. 2.

Figure 3 shows the shape of the meniscus in a few cases. As can be seen, the meniscus approaches a Taylor cone for moderate flow rates, but its volume increases and its shape becomes more elongated when the flow rate increases. These shapes are in qualitative agreement with the experimental pictures of Barrero et al., ${ }^{1}$ Marín, ${ }^{5}$ and Riboux et al. ${ }^{6}$ though in the experiments the jet is not as thick as the outermost jet in Fig. 3. The difference may be due to the values of the parameters used in the computations. According to $(14 \mathrm{c})$, the ratio $r_{s_{c t}} / a$ is of the order of $(\lambda \Lambda)^{-1 / 2}$ for $Q=Q_{M}$, which is about 0.2 in the conditions of the computations but may be of the order of $10^{-2}$ in some of the experiments of Ref. 5 . Gundabala and Fernández-Nieves ${ }^{8}$ note that thick jets (nonsmall values of $\left.r_{s_{c t}} / a\right)$ make for a slow increase of the electric current with the flow rate, as in the experiments of Riboux et $a l .{ }^{6}$ and those of Hohman et al. ${ }^{22}$ on electrospinning in air, in which the capillary number is fairly large. This may be due to the electric field acting on the current transfer region of the jet, ${ }^{23}$ as probably the field acting on a thick jet decreases with streamwise distance faster than the field induced by a conical meniscus.

The distance to the needle, $x_{\text {cross }}$, at which the surface convection and bulk conduction contributions to the electric current are equal to each other is shown in Fig. 4(a), and the radius of the jet at this point, $r_{s_{\text {cross }}}=r_{s}\left(x_{\text {cross }}\right)$, is shown in Fig. 4(b). The distance $x_{\text {cross }}$ is a measure of the extent of the current transfer region. In agreement with the first two estimates (14c), which can be rewritten as $x_{c t}^{\prime} \sim \lambda^{2} \Lambda \mathrm{Ca}$ and $r_{s_{c t}} \sim \lambda^{1 / 2} \mathrm{Ca}^{1 / 2}$ in dimensionless variables, $x_{\text {cross }}$ increases nearly linearly with the flow rate and $r_{s_{\text {cross }}}$ increases nearly as the square root of the flow rate in a range of values of this variable. The more rapid increase of $x_{\text {cross }}$ at small flow rates in some of the curves of Fig. 4(a) agrees with the prediction (14b) $\left(x_{c t}^{\prime} \sim \mathrm{Ca}^{1 / 2}\right.$ in dimensionless variables). Finally, the increase of $x_{\text {cross }}$ becomes more moderate on approaching the maximum flow rate, when the electric field acting on the current transfer region differs noticeably from that of a conical meniscus. 

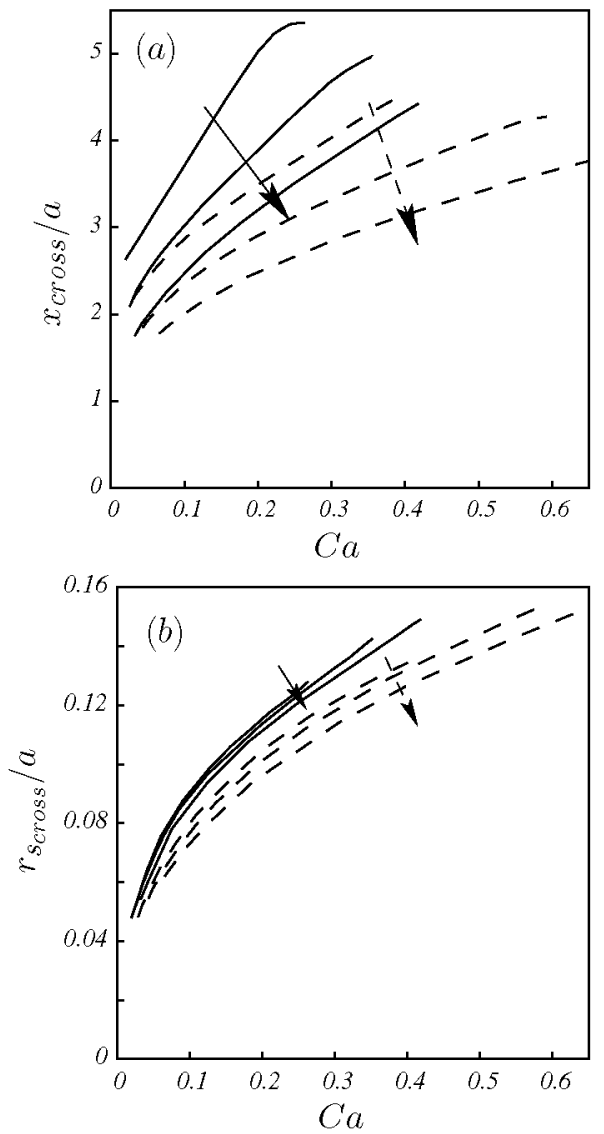

FIG. 4. Distance to the needle at which $I_{b}=I_{s}$ (a), and radius of the jet at this point (b), as functions of $\mathrm{Ca}$ for $\lambda=0.01$ (dashed curves) and 0.03 (solid curves), and $V_{\infty}=1.6,1.8$, and 2 (increasing from top to bottom as indicated be the arrows). Values of other parameters are $\epsilon=20, \Lambda=10^{3}$, and $\operatorname{Re}=0$.

The values at $x_{\text {cross }}$ of the axial forces due to the axial viscous stress in the inner liquid and to the viscous shear stress of the outer liquid on the interface [first and last terms on the right-hand side of Eq. (11), evaluated from the full numerical solution] are shown in Fig. 5 as functions of the capillary number. In line with the estimates in the paragraph

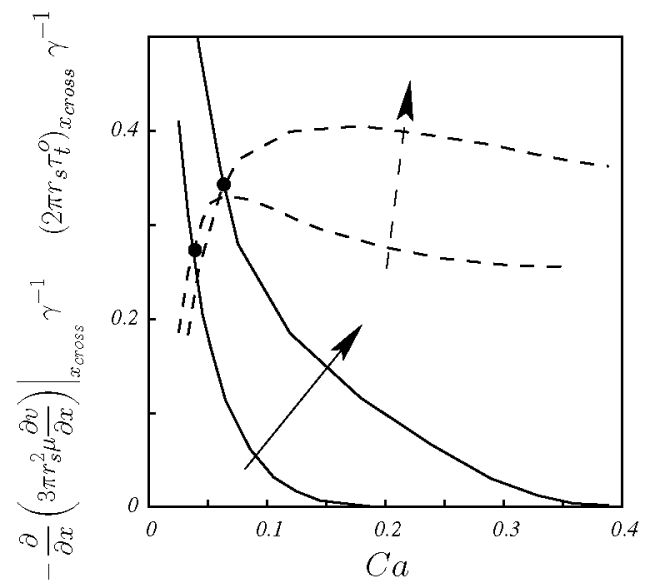

FIG. 5. Values of $-\partial\left(3 \pi r_{s}^{2} \mu \partial v / \partial x\right) / \partial x \gamma^{-1}$ (solid curves) and $2 \pi r_{s} \tau_{t}^{\rho} \gamma^{-1}$ (dashed curves), which are the first and last terms on the right-hand side of Eq. (11) nondimensionalized with $\gamma$, at $x=x_{\text {cross }}$ as functions of $\mathrm{Ca}$ for $\epsilon=20, \Lambda=10^{3}, \lambda=0.03$, and $V_{\infty}=1.8$ and 2 , increasing as indicated by the arrows.

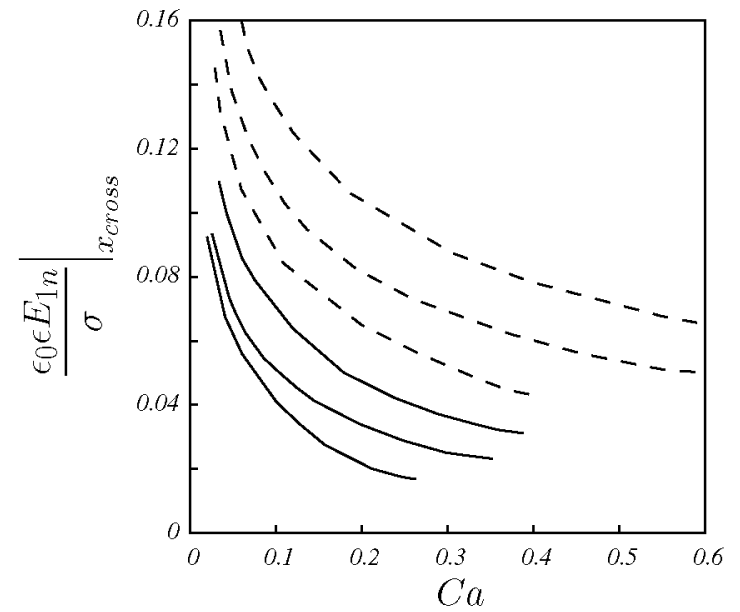

FIG. 6. Value of $\epsilon_{0} \epsilon E_{1 n} / \sigma$ at $x=x_{\text {cross }}$ as a function of Ca for $\epsilon=20$, $\Lambda=10^{3}, \lambda=0.01$ (dashed curves), and 0.03 (solid curves), and $V_{\infty}=1.6,1.8$, and 2 , increasing from bottom to top.

above (14c), the second of these forces overcomes the first [which amounts to a transition between cases (b) and (c) of the previous section] when $\mathrm{Ca}$ increases.

The ratio $\epsilon_{0} \epsilon E_{1 n} / \sigma$ at $x_{\text {cross }}$, which is a measure of the importance of charge relaxation effects in the current transfer region, is given in Fig. 6 as a function of $\mathrm{Ca}$. This figure shows that relaxation effects become important as the minimum flow rate is approached, which is generally accepted for the cone-jet mode $\mathrm{e}^{2,4,14,18}$ and supports some of the results of Sec. III C. Numerical computations showing the opposite trend for a parallel plate electrode configuration have been obtained elsewhere. ${ }^{10}$

The axial forces due to the gradient of the electrically induced depression, $\pi r_{s}^{2} \partial \tau_{n}^{e} / \partial x$ in Eq. (11), to the electric shear stress, $2 \pi r_{s} \tau_{t}^{e}$, and to the viscous drag of the outer liquid, $2 \pi r_{s} \tau_{t}^{o}$, are shown in Fig. 7 as functions of the streamwise distance for $V_{\infty}=1.8, \epsilon=20, \Lambda=10^{3}, \lambda=0.03$, and the three values of the dimensionless flow rate $\mathrm{Ca}=0.0256$, 0.125 , and 0.320 . The values of the cross-over distance are $x_{\text {cross }}=2.09,3.26$, and 4.82 , respectively, which are indicated by vertical arrows in Fig. 7. The driving force $\pi r_{s}^{2} \partial \tau_{n}^{e} / \partial x$ decreases with streamwise distance in the jet, being replaced by $2 \pi r_{s} \tau_{t}^{e}$ in the current transfer region, where the electric field tangent to the surface becomes of the order of the outer field. This changeover is pushed streamwise into the jet when Ca increases, because this increases the size of the meniscus and the length of the current transfer region. However, Fig. 7 shows that the ratio of the drag force $2 \pi r_{s} \tau_{t}^{o}$ to the force $\pi r_{s}^{2} \partial \tau_{n}^{e} / \partial x$ increases at $x_{\text {cross }}$ when Ca increases, which means that the balance of forces relies more on the electric shear stress. Apparently this balance ceases to be possible when the rise of the electric shear stress is postponed, by increasing $\mathrm{Ca}$, beyond the region where the outer field is that of a conical meniscus. No stationary solution is found for $\mathrm{Ca}$ larger than about 0.35 for the set of parameter values used in Fig. 7.

\section{CONCLUSIONS}

A qualitative analysis and numerical computations have been used to study the flow, the electric field, and the distribution of surface charge in a stationary jet of an electrically 

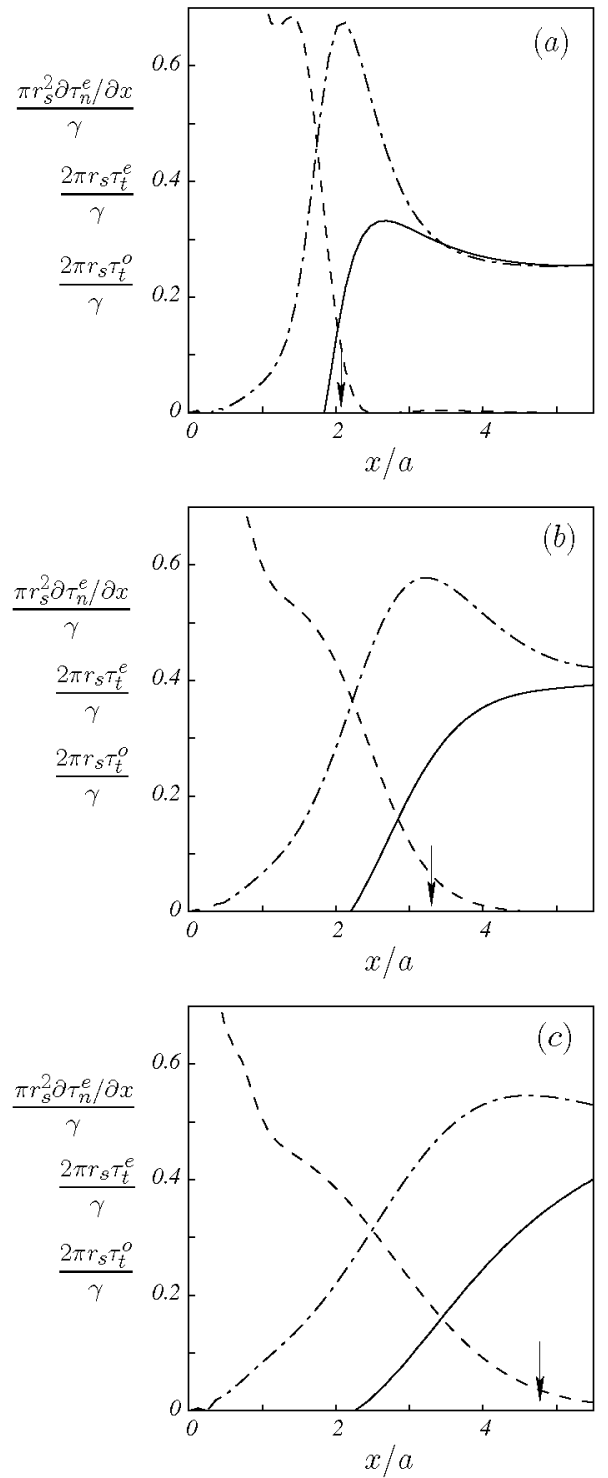

FIG. 7. Dimensionless axial forces on the jet due to the gradient of the electrically induced depression $\left(\pi r_{s}^{2} \partial \tau_{n}^{e} / \partial x \gamma^{-1}\right.$, dashed), to the electric shear stress $\left(2 \pi r_{s} \tau_{t}^{e} \gamma^{-1}\right.$, chain), and to the viscous drag of the outer liquid ( $2 \pi r_{s} \tau_{t}^{0} \gamma^{-1}$, solid), as functions of the distance to the needle for $V_{\infty}=1.8$, $\epsilon=20, \Lambda=10^{3}, \lambda=0.03, \mathrm{Re}=0$, and $\mathrm{Ca}=0.0256$ (a), 0.125 (b), and 0.350 (c). The vertical arrows mark the cross-over point $x_{\text {cross }}$.

conducting liquid injected at constant flow rate into an immiscible dielectric liquid through a metallic capillary needle held at a high voltage. The well-known square root law for the electric current as a function of the flow rate is obtained in a certain range of flow rates whose lower bound is determined by the appearance of charge relaxation effects in the current transfer region where the total current of the jet is fixed. The length of this region and the radius of the jet in it are estimated for different regimes of the flow. The electric current also departs from the square root law when the flow rate becomes of the order of a certain characteristic value, $Q_{M}$, for which the length of the current transfer region is of the order of the size of the meniscus. The electric current is predicted to level to a constant value when the viscous force of the dielectric liquid on the surface of the jet is important and the flow rate becomes large compared to $Q_{M}$. In these conditions, the length of the straight stationary jet cannot be much larger than the size of the meniscus.

\section{ACKNOWLEDGMENTS}

This work is dedicated to the memory of Professor Antonio Barrero, who introduced me to the study of electrosprays and enthusiastically explained to me his many original ideas on these problems. Support from the Spanish Ministerio de Ciencia e Innovación through Projects Nos. DPI2007-66659-C03-02 and DPI2010-20450-C03-01 is acknowledged.

${ }^{1}$ A. Barrero, J. M. López-Herrera, A. Boucard, I. G. Loscertales, and M. Márquez, "Steady cone-jet electrosprays in liquid insulator baths," J. Colloid Interface Sci. 272, 104 (2004).

${ }^{2}$ J. Fernández de la Mora, "The fluid dynamics of Taylor cones," Annu. Rev. Fluid Mech. 39, 217 (2007).

${ }^{3}$ M. S. Alexander, "Pulsating electrospray modes at the liquid-liquid interface," Appl. Phys. Lett. 92, 144102 (2008).

${ }^{4}$ J. Fernández De La Mora and I. G. Loscertales, "The current emitted by highly conducting Taylor cones," J. Fluid Mech. 260, 155 (1994).

${ }^{5}$ A. G. Marín, "Generación y dinámica de chorros electrificados. Aplicación a la síntesis de emulsiones," Ph.D. thesis, University of Seville, Spain, 2008.

${ }^{6}$ I. G. Loscertales, G. Riboux, A. G. Marín, A. Barrero, and A. FernándezNieves, "Experimental characterization of the whipping instability of charged microjets in liquid baths," in Proceedings of the 2010 MRS Spring Meeting, San Francisco, CA, 2010, Mater. Res. Soc. Symp. Proc. No. 1272, edited by C. J. Martínez et al. (MRS, Warrendale, PA, 2010), Vol. 1272, Paper No. KK05-01.

${ }^{7} \mathrm{C}$. Larriba-Andaluz and J. Fernández de la Mora, "Electrospraying insulating liquids via charged nanodrop injection from the Taylor cone of an ionic liquid," Phys. Fluids 22, 072002 (2010).

${ }^{8}$ V. R. Gundabala, N. Vilanova, and A. Fernández-Nieves, Phys. Rev. Lett. 105, 154503 (2010).

${ }^{9}$ J. Happel and H. Brenner, Low Reynolds Number Hydrodynamics (Prentice-Hall, Englewood Cliffs, 1965).

${ }^{10} \mathrm{~F}$. J. Higuera, "Numerical computation of the domain of operation of an electrospray of a very viscous liquid," J. Fluid Mech. 648, 35 (2010).

${ }^{11}$ L. D. Landau and E. M. Lifshitz, Electrodynamics of Continuous Media (Pergamon, Oxford, 1960).

${ }^{12}$ D. A. Saville, "Electrohydrodynamics: The Taylor-Melcher leaky dielectric model," Annu. Rev. Fluid Mech. 29, 27 (1997).

${ }^{13}$ G. K. Batchelor, An Introduction to Fluid Dynamics (Cambridge University Press, Cambridge, 1967).

${ }^{14}$ A. M. Gañán-Calvo, J. Dávila, and A. Barrero, "Current and droplet size in the electrospraying of liquids. Scaling laws," J. Aerosol Sci. 28, 249 (1997).

${ }^{15}$ J. J. Feng, "The stretching of an electrified non-Newtonian jet: A model for electrospinning," Phys. Fluids 14, 3912 (2002).

${ }^{16}$ G. I. Taylor, "Disintegration of water drops in an electric field," Proc. R. Soc. London, Ser. A 280, 383 (1964).

${ }^{17}$ E. J. Hinch, Perturbation Methods (Cambridge University Press, Cambridge, 1991).

${ }^{18}$ F. J. Higuera, "Flow rate and electric current emitted by a Taylor cone," J. Fluid Mech. 484, 303 (2003).

${ }^{19} \mathrm{~F}$. J. Higuera, "Stationary viscosity-dominated electrified capillary jets," J. Fluid Mech. 558, 143 (2006).

${ }^{20}$ M. B. Glauert and M. J. Lighthill, "The axisymmetric boundary layer on a long thin cylinder," Proc. R. Soc. London, Ser. A 230, 188 (1955).

${ }^{21} \mathrm{~F}$. J. Higuera, "Charge separation in the conical meniscus of an electrospray of a very polar liquid: Its effect on the minimum flow rate," Phys. Fluids 21, 032104 (2009)

${ }^{22}$ M. M. Hohman, M. Shin, G. Rutledge, and M. P. Brenner, "Electrospinning and electrically forced jets. II. Applications," Phys. Fluids 13, 2221 (2001).

${ }^{23} \mathrm{~F}$. J. Higuera, "Current/flow rate characteristic of an electrospray with a small meniscus," J. Fluid Mech. 513, 239 (2004). 